\title{
Observations of Broadband Acoustic Backscattering From Nonlinear Internal Waves: Assessing the Contribution From Microstructure
}

\author{
Andone C. Lavery, Dezhang Chu, and James N. Moum
}

\begin{abstract}
In this paper, measurements of high-frequency broadband (160-590 $\mathrm{kHz})$ acoustic backscattering from surface trapped nonlinear internal waves of depression are presented. These waves are ideal for assessing the contribution from oceanic microstructure to scattering as they are intensely turbulent. Almost coincident direct microstructure measurements were performed and zooplankton community structure was characterized using depth-resolved net sampling techniques. The contribution to scattering from microstructure can be difficult to distinguish from the contribution to scattering from zooplankton using a single narrowband frequency as microstructure and zooplankton are often colocated and can have similar scattering levels over a range of frequencies. Yet their spectra are distinct over a sufficiently broad frequency range, allowing broadband backscattering measurements to reduce the ambiguities typically associated with the interpretation of narrowband measurements. In addition, pulse compression signal processing techniques result in very high-resolution images, allowing physical processes that are otherwise hard to resolve to be imaged, such as Kelvin-Helmholtz shear instabilities. In this study, high-resolution acoustic observations of multiple nonlinear internal waves are presented and regions with distinct scattering spectra are identified. Spectra that decrease in level across the available frequency band were highly correlated to regions of intense turbulence and high stratification, and to Kevin-Helmholtz shear instabilities in particular. Spectra that increase in level across the available frequency band were consistent with scattering dominated by small zooplankton. Simple inversions for relevant microstructure parameters are presented. Limitations of, and improvements to, the broadband system and techniques utilized in this study are discussed.
\end{abstract}

Index Terms-Acoustic scattering, broadband, nonlinear internal waves, turbulent oceanic microstructure, zooplankton.

\section{INTRODUCTION}

A COUSTIC backscattering techniques provide a unique and powerful tool to remotely investigate the physical properties of the ocean interior over a large range of spatial

Manuscript received June 10, 2009; revised March 05, 2010; accepted March 17, 2010. Date of publication October 07, 2010; date of current version November 30, 2010. This work was supported in part by the Woods Hole Oceanographic Institution and the U.S. Office of Naval Research under Grant N000140210359.

Associate Editor: R. Chapman.

A. C. Lavery is with the Department of Applied Ocean Physics and Engineering, Woods Hole Oceanographic Institution, Woods Hole, MA 02543 USA (e-mail: alavery@ whoi.edu).

D. Chu is with North West Fisheries Science Center, Seattle, WA 98112 USA (e-mail: Dezhang.Chu@noaa.gov).

J. N. Moum is with the College of Oceanic and Atmospheric Sciences, Oregon State University, Corvallis, OR 97331 USA (e-mail: moum@ coas.oregonstate. edu).

Color versions of one or more of the figures in this paper are available online at http://ieeexplore.ieee.org.

Digital Object Identifier 10.1109/JOE.2010.2047814 and temporal scales. These techniques have been commonly used in the ocean to obtain highly informative, though often qualitative, images of the physical processes that occur in the ocean interior [1]-[5], including, for example, internal waves [6], [7], hydraulic jumps [8], [9], bubbles [10], Langmuir circulation [11], and microstructure [12]-[15]. Microstructure refers to fluctuations in temperature and salinity, resulting in fluctuations in sound speed and density (which in turn scatter sound), that occur at microstructure scales, that is, millimeters to tens of centimeters. Oceanic microstructure generated by stratified turbulence is the primary focus of this study, referred to throughout this study as turbulent microstructure, to distinguish it from microstructure generated by other processes, such as double diffusion.

Quantification of high-frequency acoustic backscattering from turbulent microstructure in terms of physically meaningful parameters has been a long standing challenge. There are many factors that result in ambiguities in the quantitative interpretation of acoustic backscattering from microstructure. Scattering can arise not only from microstructure, but also from zooplankton, and other biological organisms (e.g., fish). Many of these scattering sources are intermittent, and can occur simultaneously [16], [17]. Understanding the scattering from any one of these scattering sources is independently challenging as the scattering depends on many parameters [18]-[20], a number of which are difficult to quantify at relevant scales by any sampling technique, particularly in situ. For example, for zooplankton, the scattering characteristics depend in a complicated way on the material properties (sound speed and density), shape and size, orientation of the organisms relative to the incident acoustic wave, and on the acoustic frequency. For microstructure, the scattering characteristics depend on parameters such as the temperature and salinity gradients, the dissipation rate of turbulent kinetic energy, the degree of anisotropy, as well as the acoustic frequency [21]. Adding to these difficulties, scattering from zooplankton and oceanic microstructure can have similar scattering levels at many commonly used (e.g., 120, 200, and $420 \mathrm{kHz}$ ) frequencies. Further compounding the complications, high-frequency acoustic backscattering measurements are typically performed at a single narrowband frequency, or a small number of narrowband frequencies, limiting the amount of information available for interpretation of the scattering measurements. Generally, an insufficient number of discrete narrowband frequencies are used to accurately map the scattered spectra from the different scattering sources [20].

In this study, measurements of high-frequency broadband acoustic backscattering from microstructure and zooplankton have been made in the presence of surface trapped nonlinear 


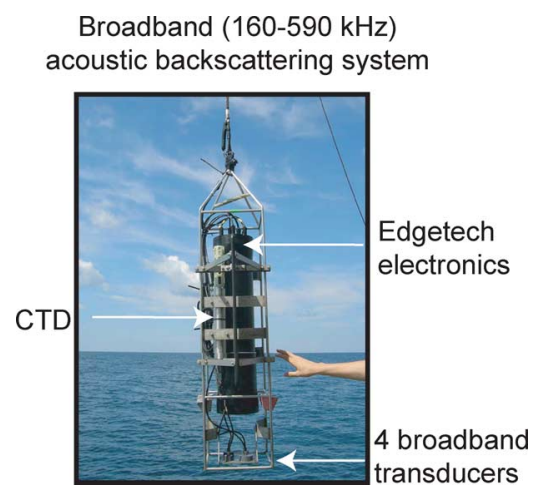

Fig. 1. Photograph of the high-frequency broadband $(160-590 \mathrm{kHz})$ acoustic backscattering system.

internal waves of depression propagating across the New Jersey continental shelf. High-resolution images of many internal wave trains have been obtained at different stages of their evolution. Almost coincident direct microstructure measurements were performed and zooplankton community structure was characterized using depth-resolved net-sampling techniques. The combination of these data results in more accurate interpretation of the acoustic scattering measurements and, in particular, the ability to determine the relative contribution to scattering from turbulent microstructure and zooplankton.

Surface trapped nonlinear internal solitary waves of depression are a unique feature of coastal oceans and provide a good environment to assess the contribution to backscattering from oceanic microstructure as they are both intensely turbulent [7] and at sufficiently close range that they generate high signal levels well within the range of surface-deployed, high-frequency broadband acoustic backscattering systems. Surface trapped nonlinear internal waves of depression are thought to be generated near the continental shelf break due to the interaction of internal tides with stratified fluid over sharp topography. They then propagate across the continental shelf until they dissipate in shallower waters. Not all the internal wave energy is dissipated in shallow waters, as some is dissipated by the generation of turbulence along the propagation path. Mechanisms for this dissipation of energy are discussed by Moum et al. [7]. It is hoped that quantification of broadband acoustic backscattering from microstructure generated by these nonlinear internal waves may ultimately contribute to understanding these dissipation mechanisms.

One of the overarching goals of this work is to use high-frequency broadband $(160-590 \mathrm{kHz})$ acoustic backscattering techniques, capitalizing on the development of a new broadband backscattering system (Fig. 1), to enhance the ability to discriminate between, and decrease the ambiguities associated with the quantitative interpretation of, acoustic scattering from microstructure and zooplankton. Though the focus of this study is oceanic microstructure, simultaneously understanding the confounding contribution to scattering from zooplankton is critical for correctly interpreting the data. The frequency range used in this study encompasses many of the narrowband acoustic frequencies typically used to survey zooplankton and turbulent oceanic microstructure (e.g., 200 and $420 \mathrm{kHz}$ ), and includes the Rayleigh-to-geometric scattering transition of some typical

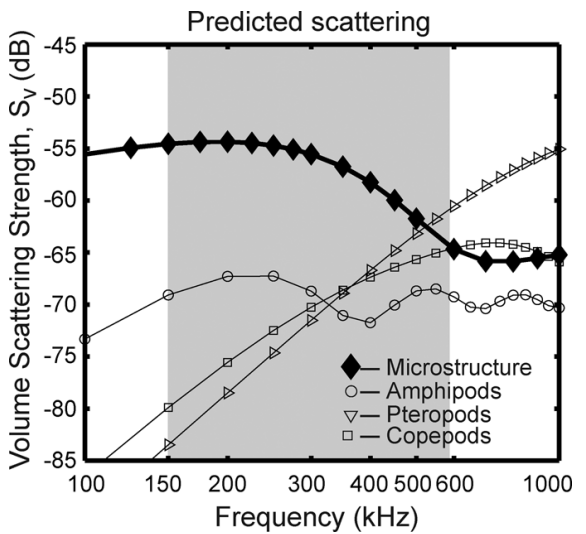

Fig. 2. Predictions of scattering from zooplankton and turbulent microstructure. Elastic-shelled pteropods and weakly scattering fluid-like copepods and amphipods dominated the predicted scattering during SW06. The scattering models used for these predictions are described in [20]. The parameters for the zooplankton predictions are based on net tow 4 and the microstructure parameters are based on values obtained for internal wave Mika 1.

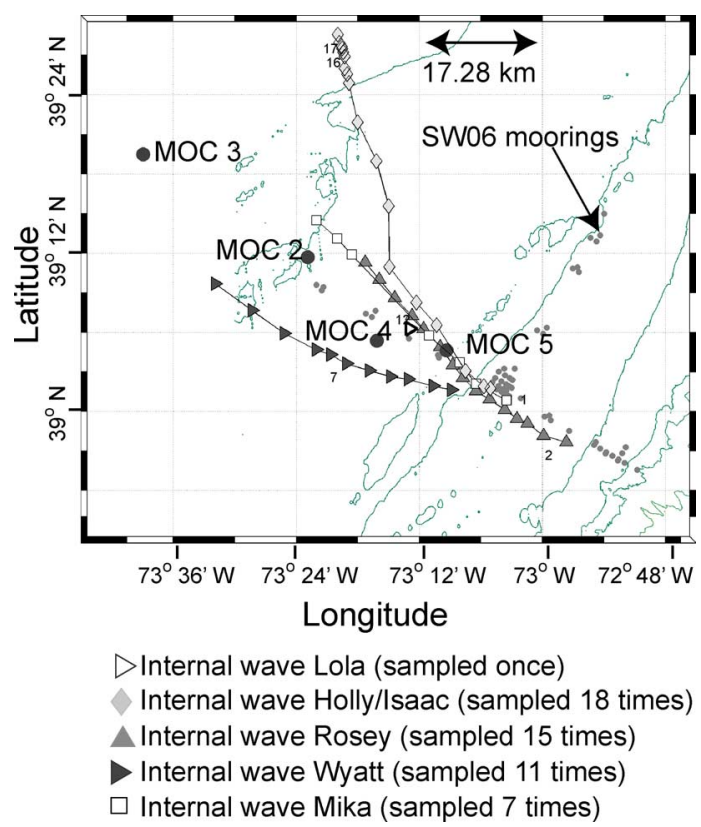

Fig. 3. Location of the SW06 experiment. The tracks of selected nonlinear internal waves are shown, superimposed on the local bathymetry. The positions of the zooplankton net tows are indicated.

zooplankton and the diffusive rolloff in the spectrum for scattering from turbulent temperature microstructure for a range of dissipation rates (Fig. 2).

In addition to allowing the scattering spectrum to be determined, broadband acoustic backscattering measurements enable the use of pulse compression signal processing techniques to obtain very high-resolution images of the nonlinear internal waves. In combination with the high ping rate $(1 \mathrm{~Hz})$, these techniques have allowed physical processes, such as Kelvin-Helmholtz shear instabilities [7], [22] typically not well resolved by the direct microstructure measurements, to be imaged at very high resolution. Though the direct microstructure measurements provide very high-resolution measurements in the vertical, the profiles are relatively sparse (one profile every 2-4 min) relative to the acoustic measurements or the 
(a) Temperature
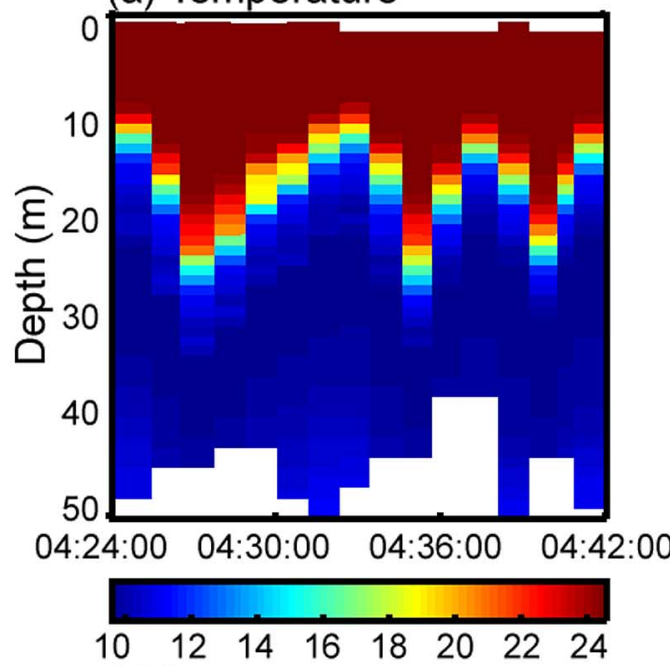

(c) $\log _{10} \varepsilon$

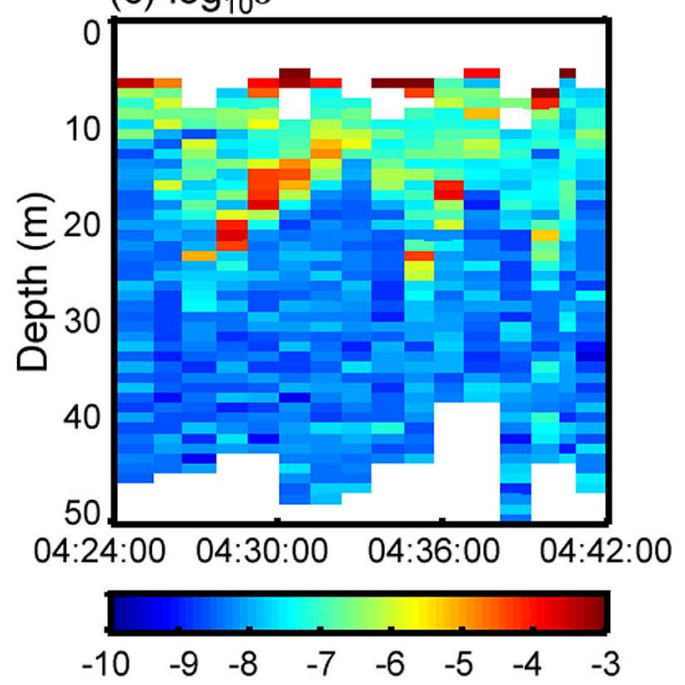

(b) Salinity

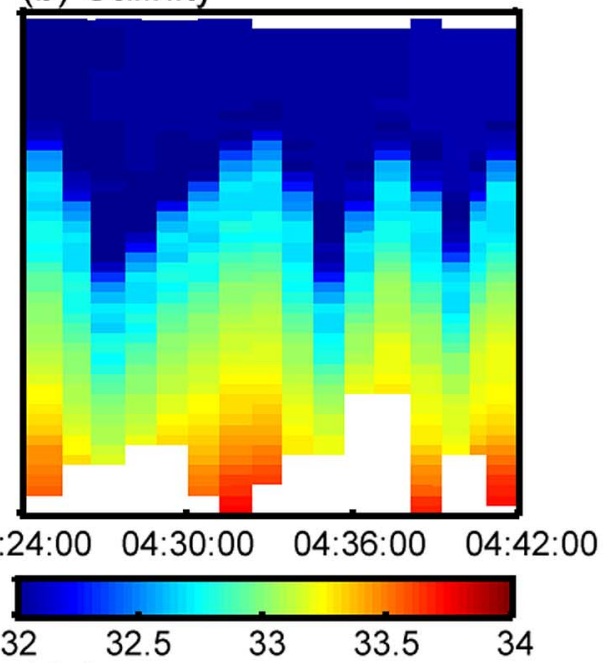

(d) $\log _{10} \chi_{T}$

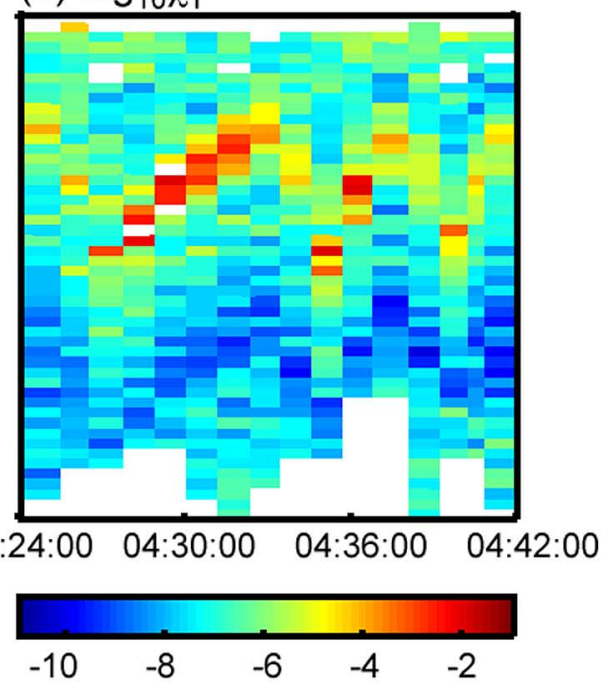

\section{Local Time (14 August 2006)}

Fig. 4. Direct microstructure measurements for nonlinear internal wave Mika 1 on August 14, 2006. (a) Temperature in degree Celsius, (b) salinity, (c) dissipation rate of turbulent kinetic energy $\varepsilon\left(\mathrm{m}^{2} \cdot \mathrm{s}^{-3}\right)$, on a logarithmic scale, and (d) dissipation rate of temperature variance $\chi_{T}\left(\mathrm{~m}^{2} \cdot \mathrm{s}^{-1}\right)$, on a logarithmic scale.

spatial scales of the Kelvin-Helmholtz instabilities. It has been found that generally decreasing scattering spectral levels over the available frequency band are strongly correlated to the occurrence of these Kelvin-Helmholtz instabilities. Simple least squares inversions of the broadband backscattering data in these instabilities have been performed allowing the dissipation rate of turbulent kinetic energy and temperature variance to be deduced at finer scales than was possible based on the direct microstructure measurements.

\section{METHODS}

\section{A. Experiment Location}

The high-frequency broadband acoustic scattering data presented here were collected during the generation, propagation, and dissipation stages of nonlinear internal waves observed over the New Jersey continental shelf (Fig. 3) from July 29, 2006 to August 27, 2006, from the $R / V$ Oceanus. These measurements were part of the U.S. Navy funded 2006 Shallow Water (SW06) acoustics and nonlinear internal waves experiment [23].

During this experiment, the water column was strongly stratified, resulting in large temperature gradients (typical temperatures reaching $22^{\circ}-25^{\circ}$ in the upper mixed layer and $8^{\circ}-12^{\circ}$ below) occurring at the depth of the seasonal thermocline at approximately 10-20 m. The passage of the nonlinear internal waves displaced these isotherms downwards, by as much as $20 \mathrm{~m}$. Salinity gradients were not as pronounced as the temperature gradients (Fig. 4). High acoustic backscattering levels often closely tracked isopycnal displacements.

The typical mode of operation involved the use of an uncalibrated, single-narrowband-frequency, hull-mounted, $120-\mathrm{kHz}$ echosounder to locate internal solitary waves offshore, which were then followed as they propagated onshore across the continental shelf. In addition to the single-frequency echosounder, a $300-\mathrm{kHz}$ acoustic Doppler current profiler (ADCP) was used to continuously measure velocities associated with the 
TABLE I

PREDicted SCATTERING From ZoOPLANKTON BASED ON THE NET TOWS WaS GeNERALLY DOMINATED BY COPEOPODS, PTEROPODS, AND AMPHIPODS. THE MEAN LENGTHS $\langle\mathrm{L}\rangle$ AND STANDARD DEVIATIONS OF LENGTHS $\sigma_{\mathrm{L}}$ FOR THESE ORGANISMS ARE GIVEN HERE FOR EACH MOCNESS TOW, AVERAGED OVER All DePTHS. THE ABUNDANCE A abund VARIED SigNIFICANTLY ACCORDING TO DEPTH AND ONLY THE MAXIMUM ABUNDANCE A max MEASURED FOR EACH MOCNESS TOW, WHICH CAN OCCUR AT A DIFFERENT DEPTH FOR Each Tow, Is Given Here. For More Details SeE [15].

\begin{tabular}{|c|c|c|c|c|}
\hline & MOC 2 & MOC 3 & MOC 4 & MOC 5 \\
\hline Copepods: $<\mathrm{L}>$ & $0.60 \mathrm{~mm}$ & $0.74 \mathrm{~mm}$ & $1.16 \mathrm{~mm}$ & $1.2 \mathrm{~mm}$ \\
\hline$\sigma_{\mathrm{L}}$ & $0.32 \mathrm{~mm}$ & $0.47 \mathrm{~mm}$ & $0.83 \mathrm{~mm}$ & $0.8 \mathrm{~mm}$ \\
\hline$A_{\max }$ & $7260 / \mathrm{m}^{3}$ & $2785 / \mathrm{m}^{3}$ & $4200 / \mathrm{m}^{3}$ & $1526 / \mathrm{m}^{3}$ \\
\hline Pteropods: $<\mathrm{L}>$ & $0.34 \mathrm{~mm}$ & $0.35 \mathrm{~mm}$ & $0.38 \mathrm{~mm}$ & $0.46 \mathrm{~mm}$ \\
\hline$\sigma_{\mathrm{L}}$ & $0.12 \mathrm{~mm}$ & $0.19 \mathrm{~mm}$ & $0.07 \mathrm{~mm}$ & $0.2 \mathrm{~mm}$ \\
\hline $\mathrm{A}_{\max }$ & $333 / \mathrm{m}^{3}$ & $28 / \mathrm{m}^{3}$ & $942 / \mathrm{m}^{3}$ & $99 / \mathrm{m}^{3}$ \\
\hline Amphipods: $<\mathrm{L}>$ & $4.8 \mathrm{~mm}$ & $4.07 \mathrm{~mm}$ & $4.36 \mathrm{~mm}$ & $3.75 \mathrm{~mm}$ \\
\hline$\sigma_{\mathrm{L}}$ & $1.7 \mathrm{~mm}$ & $2.79 \mathrm{~mm}$ & $2.03 \mathrm{~mm}$ & $2.48 \mathrm{~mm}$ \\
\hline $\mathrm{A}_{\max }$ & $113 / \mathrm{m}^{3}$ & $4 / \mathrm{m}^{3}$ & $26 / \mathrm{m}^{3}$ & $121 / \mathrm{m}^{3}$ \\
\hline
\end{tabular}

nonlinear internal waves. A broadband acoustic scattering system and microstructure profiler (described in more detail later) were deployed with the vessel slowly drifting and the internal waves were sampled as they passed by the vessel. Once the internal wave passed by, the instruments were recovered, the vessel moved rapidly ahead of the internal wave, and the instruments were redeployed. Thus, the same internal wave train was sampled on multiple occasions as it propagated across the continental shelf (Fig. 3). Twenty five internal wave trains were sampled acoustically, and each internal wave was named. Examples from nonlinear internal waves Holly/Isaac, Mika, and Wyatt are presented here. The number after the internal wave name refers to the instance in time that it was sampled. For example, Wyatt 7 refers to the seventh instance in which the internal wave train Wyatt was sampled with the broadband acoustic backscattering system and the direct microstructure system.

\section{B. Acoustic Methods}

Relatively straightforward custom modifications to a commercially available Edgetech sidescan sonar system, described in detail in [15], have made it possible to measure acoustic backscattering almost continuously over the frequency range from 160 to $590 \mathrm{kHz}$ [15]. Pertinent details of this system are presented here, together with a summary of the analysis techniques used. Details of the scattering model used for turbulent oceanic microstructure are also presented.

1) Broadband Acoustic Backscattering System: The Edgetech high-frequency broadband acoustic backscattering system (Fig. 1) has four broadband transducers with almost overlapping frequency bands, referred to as the LOW (160-270 kHz), MID (220-330 kHz), High-Low (HL: 330-470 kHz), and High-High (HH: 450-590 kHz) channels. The beamwidths vary with channel and frequency, but the half-beamwidths at center frequency were approximately $3.5^{\circ}-5.5^{\circ}$, corresponding to a footprint diameter of approximately $7 \mathrm{~m}$ at a range of $50 \mathrm{~m}$. The system also records global positioning system (GPS) time, latitude, and longitude, and pitch, roll, and heading. An integrated conductivity-temperature-depth (CTD) sensor (Seabird SBE49 sampling at $16 \mathrm{~Hz}$ ) provided measurements of temperature, conductivity, and pressure. The broadband acoustic data presented here were obtained with the system suspended close to the surface with the transducers facing downward.

The transmit signals used in this study involve linear frequency-modulated sweeps (or chirps) of 5-ms duration. The transmit sequence was LOW, MID, HL, LOW, MID, HH, with approximately $333 \mathrm{~ms}$ between channel transmissions. The typical ping rate for this entire sequence was thus $0.5 \mathrm{~Hz}$ : the LOW and MID channels sampled the water column at $1 \mathrm{~Hz}$, while the $\mathrm{HL}$ and $\mathrm{HH}$ channels sampled the water column at $0.5 \mathrm{~Hz}$. Data were collected to a range of $50 \mathrm{~m}$. The system was calibrated both before and after the experiment using standard target techniques [24]. Details of the calibration are provided in [15].

2) Processing and Analysis of Broadband Backscattering Returns: The broadband capabilities of the system are exploited through pulse-compression signal processing techniques [25]-[27], which are based on matched-filter processing, and involve cross-correlating the return echo-voltage time series $v_{r}(t)$, and the transmitted signal voltage time series $v_{t}(t)$ (which is used as the replica signal)

$$
c p_{r}(t)=k_{c p} v_{r}(t) \otimes v_{t}(t)
$$

where $\otimes$ represents cross correlation, $c p_{r}$ is the compressedpulse output, and the normalization constant $k_{c p}$ is the inverse of the autocorrelation function of $v_{t}(t)$, evaluated at zero time lag. This type of processing results in significantly increased temporal (and hence spatial) resolution and increased signal-tonoise ratios (SNRs) [25]-[27].

The volume backscattering coefficient as a function of frequency, for multiple unresolved targets in the beam, or for a distributed scattering source, is given by (modified form of [27, eq. (18)])

$$
s_{\mathrm{v}}(\omega)=G \frac{\left\langle\left|C P_{r}^{\mathrm{vol}}(\omega)\right|^{2}\right\rangle}{\left\langle\left|C P_{r 0}^{\mathrm{cal}}(\omega)\right|^{2}\right\rangle}\left|\frac{L^{\mathrm{cal}}(\omega)}{L^{\mathrm{vol}}(\omega)}\right|^{2} \frac{\sigma_{b s}^{\mathrm{cal}}(\omega)}{V(\omega)}
$$

where $\omega$ is the angular frequency, $G$ is the ratio of the transmit power during calibration and actual transmission, $\left|C P_{r}^{\mathrm{vol}}(\omega)\right|$ is the absolute magnitude of the Fourier transform of the compressed-pulse output of the volume of interest, $\left|C P_{r 0}^{c a l}(\omega)\right|$ is the absolute magnitude of the Fourier transform of compressed-pulse output obtained during the standard target calibration, and averages, denoted by $\langle\ldots\rangle$, are taken over a number of pings (typically ten). $\sigma_{b s}^{\text {cal }}(\omega)$ is the theoretically predicted backscattering cross section, based on the modal series solution, of the calibration standard target. $V(\omega)$ is the frequency-dependent equivalent insonified volume, and $L^{\mathrm{vol}}(\omega)$ and $L^{\mathrm{cal}}(\omega)$ represent the transmission loss for the scattered volume of interest and the calibration signals attributable to 


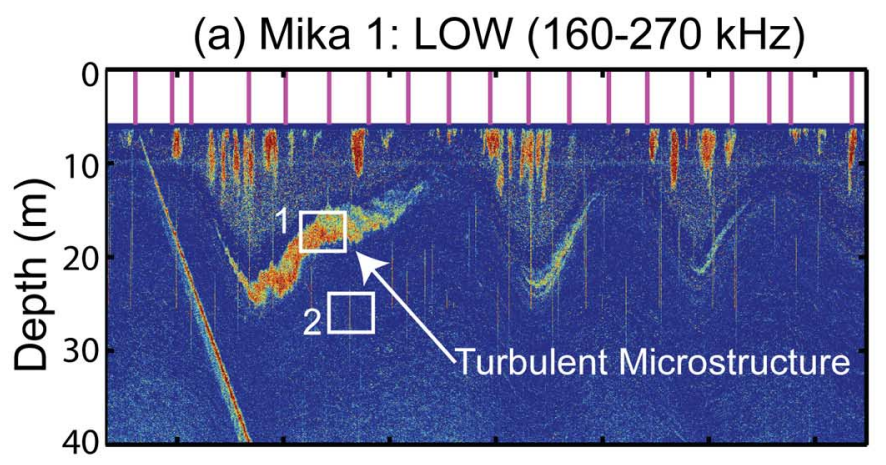

(b) Mika 1: HH (450-590 kHz)
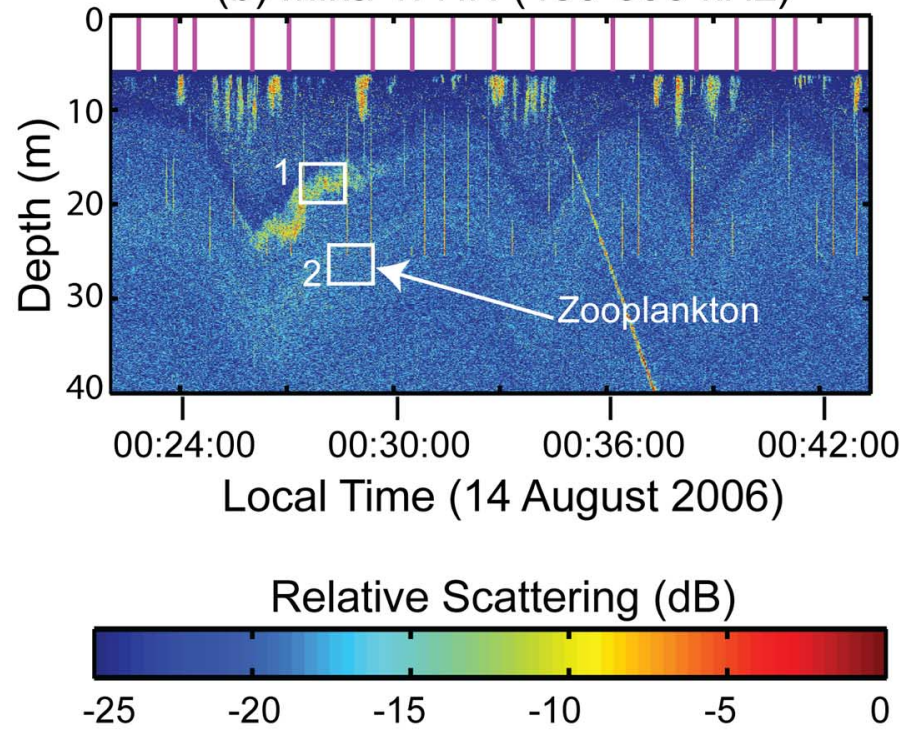

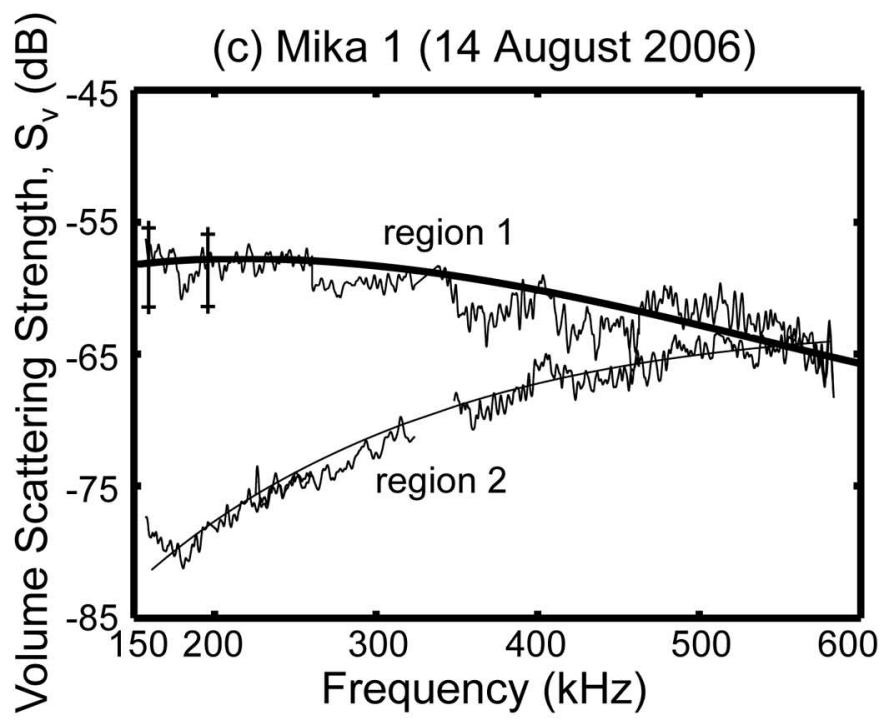

Fig. 5. Compressed pulse output as a function of depth and local time for nonlinear internal wave Mika 1 on August 14, 2006: (a) LOW and (b) HH frequency channels. (c) Typical scattering spectra showing volume scattering strength (with units of decibels relative to $1 \mu$ Pa at $1 \mathrm{~m}$ ) versus frequency. The theoretical scattering predictions (solid lines) are based on the results of the inversion for dissipation rates of turbulent kinetic energy $\left(\varepsilon=8 \times 10^{-6} \mathrm{~m}^{2} \cdot \mathrm{s}^{-3}\right)$ and temperature variance $\left(\chi_{T}=3 \times 10^{-3} \mathrm{~m}^{2} \cdot \mathrm{s}^{-1}\right)$ in region 1 , and pteropods abundance (61 individuals per cubic meter) and diameter $(0.78 \mathrm{~mm})$ in region 2 . Errors bars represent one std of the spectral noise, and are representative of the error bars throughout this study. For details of the analysis leading to the compressed pulse images, see [15]. The same color bar, which represents a relative scale, is used for all compressed pulse output images in this study, and the vertical lines at the top of the compressed pulse images show the times of the direct microstructure profiles. The occasional strong diagonal traces, and weaker vertical traces, are noise spikes picked up from an unknown source.

spherical spreading and absorption. The volume backscattering strength $S_{\mathrm{v}}=10 \log _{10} s_{\mathrm{v}}$ is the logarithmic equivalent of (2) in decibels relative to $1 \mu \mathrm{Pa}$ at $1 \mathrm{~m}$, and is calculated for each channel independently.

3) Scattering Models: The acoustic scattering models used to predict scattering from zooplankton and turbulent oceanic microstructure are described in detail in [20]. The necessary parameters for making predictions using these scattering models can be obtained from the direct microstructure measurements and zooplankton net tows. The scattering model used to describe scattering from homogeneous and isotropic turbulent oceanic microstructure is reproduced here due to its importance in this study. The volume backscattering coefficient (with units of inverse meter) for turbulent microstructure is given by [21]

$$
s_{\mathrm{v}}=2 \pi k^{4}\left(A^{2} \Phi_{T}(K)+B^{2} \Phi_{S}(K)+2 A B \Phi_{T S}(K)\right)
$$

where $k$ is the acoustic wave number $(k=2 \pi / \lambda$ where $\lambda$ is the acoustic wavelength) and $K=2 k$ is the Bragg wave number in the backscattering direction, and $\Phi_{T}(K), \Phi_{S}(K)$, and $\Phi_{T S}(K)$, are the 3-D wave number spectra of temperature, salinity, and the temperature-salinity cospectrum, all evaluated at the Bragg wave number $K$. The first term represents scattering from turbulent temperature microstructure, the second terms represents scattering from turbulent salinity microstructure, and the last term represents scattering from the cospectrum of temperature and salinity microstructure. The parameters $A$ and $B$ are defined as $A=c^{-1}(\partial c / \partial T)+\rho^{-1}(\partial \rho / \partial T)$ and $B=c^{-1}(\partial c / \partial S)+\rho^{-1}(\partial \rho / \partial S)$, where $c$ is sound speed $\left(\mathrm{m} \cdot \mathrm{s}^{-1}\right), T$ is temperature (in degrees Celsius), $S$ is salinity, and $\rho$ is density $\left(\mathrm{kg} \cdot \mathrm{m}^{-3}\right)$.

Assuming that the turbulent microstructure is accurately described as homogeneous and isotropic, then the volume backscattering coefficient can be expressed in terms of the $1-\mathrm{D}$ wave number spectra, which is what is measured by the microstructure profiling instrument

$$
s_{\mathrm{v}}=\frac{k^{4}}{K}\left(A^{2} \frac{d \phi_{T}(K)}{d k}+B^{2} \frac{d \phi_{S}(K)}{d k}+2 A B \frac{d \phi_{T S}(K)}{d k}\right) \text {. }
$$


(a) Holly 17/ Isaac 10: LOW (160-270 kHz)
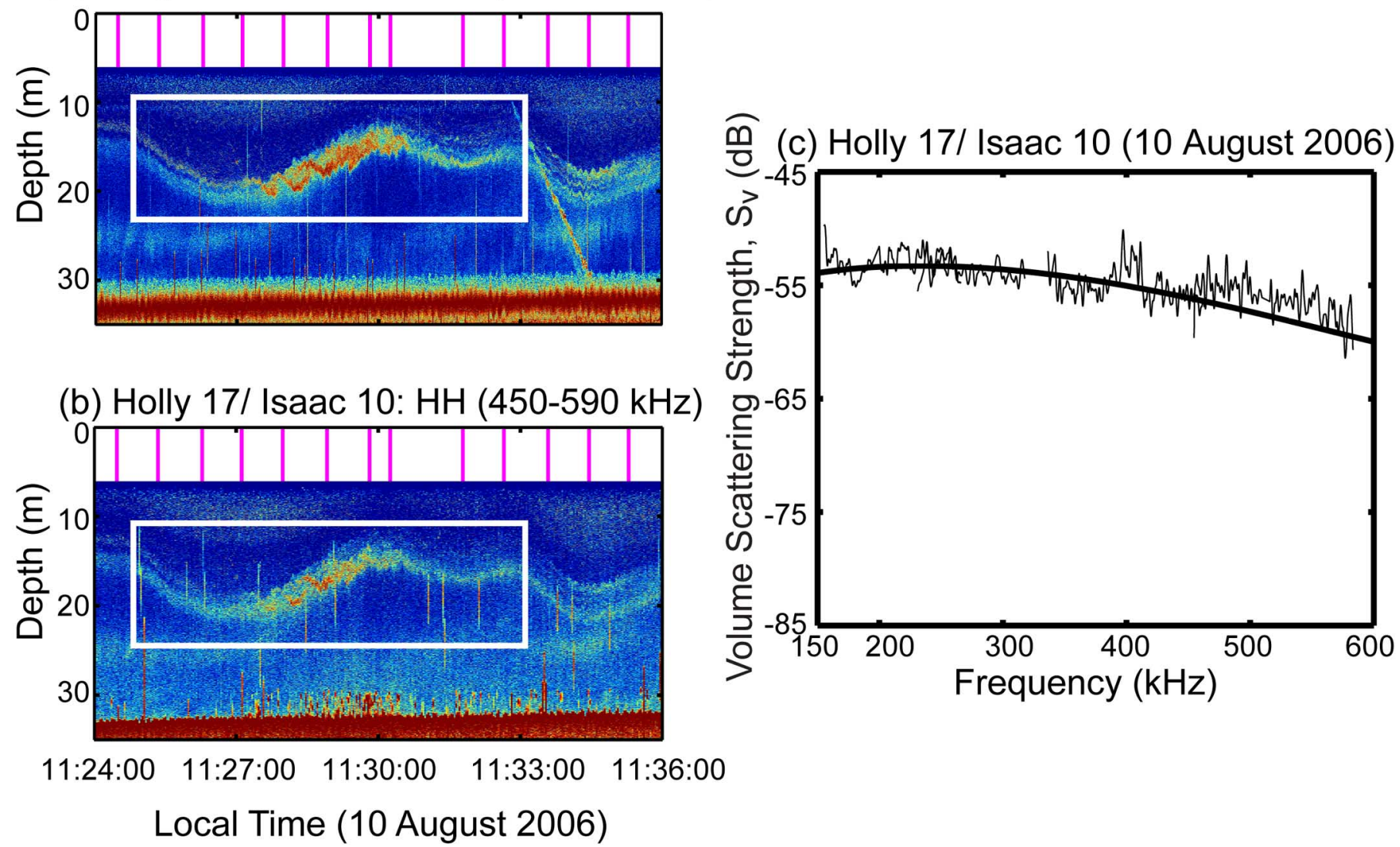

Fig. 6. Compressed pulse output as a function of depth and local time for nonlinear internal wave Holly 17/Isaac 10 imaged on August 10, 2006. (a) LOW and (b) HH frequency channels. A typical decreasing scattering spectrum is shown for the shear instability observed during the passage of this internal wave. The theoretical scattering prediction is based on the results of the inversion for dissipation rates of turbulent kinetic energy $\left(\varepsilon=4 \times 10^{-5} \mathrm{~m}^{2} \cdot \mathrm{s}^{-3}\right)$ and temperature variance $\left(\chi_{T}=1 \times 10^{-2} \mathrm{~m}^{2} \cdot \mathrm{s}^{-1}\right)$ in the shear instability. Inversions for dissipation rate of turbulent kinetic energy and temperature variance are performed throughout the region enclosed by the white box.

At the frequencies of interest here the observed scattering is in the dissipation subrange, and a Batchelor spectrum can be used to describe the temperature and salinity spectra, with the cospectrum model proposed in [28]

$$
\begin{aligned}
s_{\mathrm{v}}=\left(\frac{k^{4}}{K^{3}}\right) q\left(\frac{\nu}{\varepsilon}\right)^{1 / 2}\{ & A^{2} \chi_{T} e^{-q\left(K^{2} / k_{B T}^{2}\right)} \\
& +B^{2} \chi_{S} e^{-q\left(K^{2} / k_{B S}^{2}\right)} \\
& \left.+A B\left(\chi_{T} \chi_{S}\right)^{1 / 2} e^{-q\left(K^{2} / k_{B T S}^{2}\right)}\right\}
\end{aligned}
$$

where $\varepsilon$ is the dissipation rate of turbulent kinetic energy $\left(\mathrm{m}^{2}\right.$. $\left.\mathrm{s}^{-3}\right), \nu$ is the molecular viscosity $\left(\mathrm{m}^{2} \cdot \mathrm{s}^{-1}\right)$, and $q=3.7$ is a universal constant [29]. $k_{B T}=\left(\varepsilon /\left(\nu \kappa_{T}^{2}\right)\right)^{1 / 4}, k_{B S}=$ $\left(\varepsilon /\left(\nu \kappa_{S}^{2}\right)\right)^{1 / 4}$, and $k_{B T S}=\left(\varepsilon /\left(\nu \kappa_{T S}^{2}\right)\right)^{1 / 4}$, where $\kappa_{T}\left(\mathrm{~m}^{2}\right.$. $\left.\mathrm{s}^{-1}\right)$ and $\kappa_{S}\left(\mathrm{~m}^{2} \cdot \mathrm{s}^{-1}\right)$ are the molecular diffusivities for temperature and salt, and $\kappa_{T S}=0.5\left(\kappa_{T}+\kappa_{S}\right)$. The dissipation rates of temperature and salinity variance are given by $\chi_{T}\left(\mathrm{~m}^{2} \cdot \mathrm{s}^{-1}\right)$ and $\chi_{S}\left(\mathrm{~m}^{2} \cdot \mathrm{s}^{-1}\right)$.

Assuming that temperature and salinity have equal eddy diffusivities, it follows that $\chi_{S}$ is equal to $\chi_{T}$ scaled by the square of the ratio of the vertical salinity gradient to the vertical temperature gradient. Throughout much of this study, the temperature gradients were significantly larger than the salinity gradients, and thus $\chi_{S} / \chi_{T} \ll 1$. As a result, the predicted scattering was often dominated by temperature microstructure. However, it is important to include the contributions to scattering from salinity microstructure as scattering due to temperature microstructure alone does not accurately reproduce the observed scattering at frequencies above the temperature microstructure rolloff. The frequency at which the temperature dissipation spectrum rolls off is determined by $\varepsilon$, and for many values of $\varepsilon$ in this study, there was an observable rolloff in the frequency band available, and for frequencies above the rolloff, the scattering from microstructure decreases with increasing frequency. In contrast, the absolute scattering levels are determined by a combination of the temperature and salinity gradients, and hence by $\chi_{T}$ and $\chi_{S}$, and by $\varepsilon$. Thus, a larger value of $\varepsilon$ does not necessarily correspond to higher scattering levels.

4) Acoustic Scattering Spectra: Acoustic scattering spectra, defined as volume scattering strength versus acoustic frequency, were calculated for a number of different nonlinear internal waves. A depth bin of $1 \mathrm{~m}$ was typically used for calculating acoustic spectra, chosen to match the 1-m depth bins over which turbulent kinetic energy dissipation rates were calculated from the direct microstructure measurements. The spectra from individual pings were averaged over a 10 -s time period. The nonlinear internal waves travel at approximately $0.5-1 \mathrm{~m} \cdot \mathrm{s}^{-1}$, 
(a) Wyatt 7: LOW (160-270 kHz)
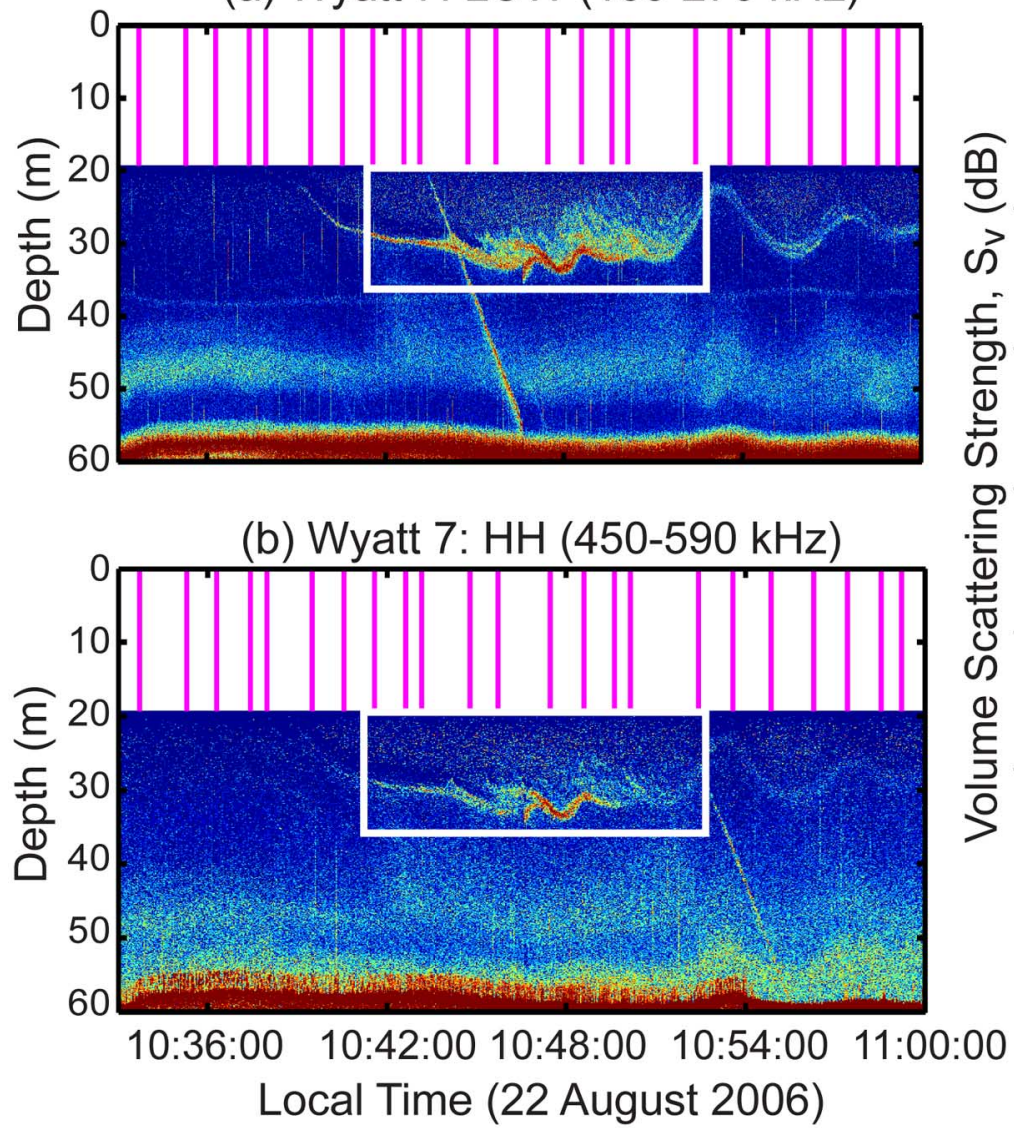

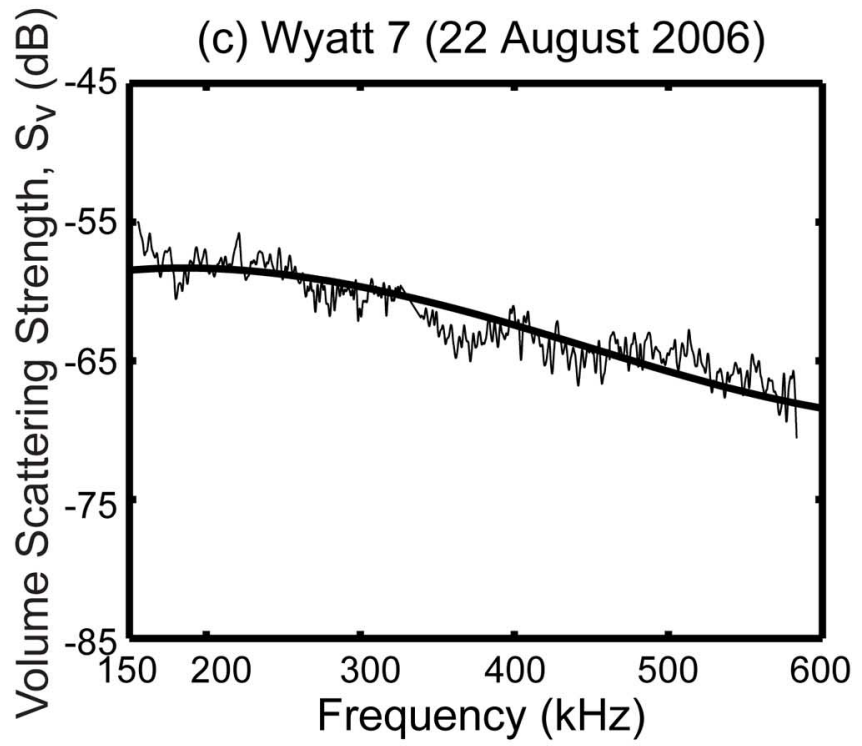

Fig. 7. Compressed pulse output as a function of depth and local time for nonlinear internal wave Wyatt 7 imaged on August 22 , 2006. (a) LOW and (b) HH frequency channels. A typical decreasing scattering spectrum is shown in (c) for the shear instability observed during the passage of this internal wave. The theoretical scattering prediction is based on the results of the inversion for dissipation rates of turbulent kinetic energy $\left(\varepsilon=4 \times 10^{-6} \mathrm{~m}^{2} \cdot \mathrm{s}^{-3}\right)$ and temperature variance $\left(\chi_{T}=2.5 \times 10^{-4} \mathrm{~m}^{2} \cdot \mathrm{s}^{-1}\right)$ in the shear instability. Inversions for dissipation rates of turbulent kinetic energy and temperature variance have been performed throughout the region enclosed by the white box.

thus a 10-s average corresponds to a horizontal spatial scale of approximately $5-10 \mathrm{~m}$.

For some of the analyses, it was useful to categorize the acoustic spectra into the following three general types:

1) Increasing: $\left.\left(\left\langle\mathrm{S}_{\mathrm{V}}^{\mathrm{HH}}\right\rangle-\left\langle\mathrm{S}_{\mathrm{V}}^{\mathrm{LOW}}\right\rangle\right)\right\rangle 3 \sigma_{\mathrm{STD}}$ where $\langle\cdots\rangle$ represents the mean over the frequency band, and $\sigma_{\mathrm{STD}}$ represents the standard deviation (std) of the spectrum noise, which was typically around $2 \mathrm{~dB}$.

2) Decreasing: $\left(\left\langle\mathrm{S}_{\mathrm{V}}^{\mathrm{LOW}}\right\rangle-\left\langle\mathrm{S}_{\mathrm{V}}^{\mathrm{HH}}\right\rangle\right)>3 \sigma_{\mathrm{STD}}$.

3) Other: Any spectra that did not fall into the previous categories, including spectra that were "flat" or did not rise above the measured noise threshold.

To understand acoustic scattering from zooplankton, it is convenient to categorize zooplankton into three groups based on the boundary conditions and anatomy [18]-[20]: 1) weakly scattering fluid-like zooplankton, for which the density and soundspeed contrasts are small relative to the surrounding water; 2) zooplankton with hard elastic shells, which scatter sound very efficiently relative to fluid-like zooplankton of similar size; and 3) zooplankton with gas inclusions (not observed in significant abundance in this study) that can give rise to strong scattering resonances, in much the same way as scattering from bubbles or fish. The vast majority of zooplankton biomass and numerical abundance is made up of fluid-like zooplankton, typically small copepods. Acoustic scattering spectra for fluid-like and elastic-shelled zooplankton increase rapidly with increasing frequency below the Rayleigh-to-geometric scattering transition (defined as $k a=1$, where $a$ is a typical dimension of the scatterer, such as width) before approximately leveling off.

Over the frequency band available in this study, the scattering spectrum for small nongas-bearing zooplankton (smaller than approximately $2 \mathrm{~mm}$ in length) is expected to increase rapidly with frequency, as the scattering has not yet reached the Rayleigh-to-geometric scattering transition. However, the frequency dependence of larger nongas-bearing zooplankton could range from slowly increasing to generally flat, but not decreasing significantly, across the frequency band of interest. In contrast, as discussed above, the shape of the acoustic scattering spectrum for turbulent microstructure could range anywhere from approximately flat to sharply decreasing with increasing frequency, depending on the relative temperature and salinity gradients and $\varepsilon$. Thus, though the classification of a flat spectrum is highly ambiguous, a generally decreasing spectrum over the available frequency band, in the absence of small gas-bearing zooplankton, is consistent with turbulent oceanic microstructure (Fig. 2).

5) Acoustic Inversions: Simple least squares inversions for size and abundance of zooplankton were performed in regions where the acoustic spectra were categorized as increasing across the full bandwidth available (see above spectral classifications). 


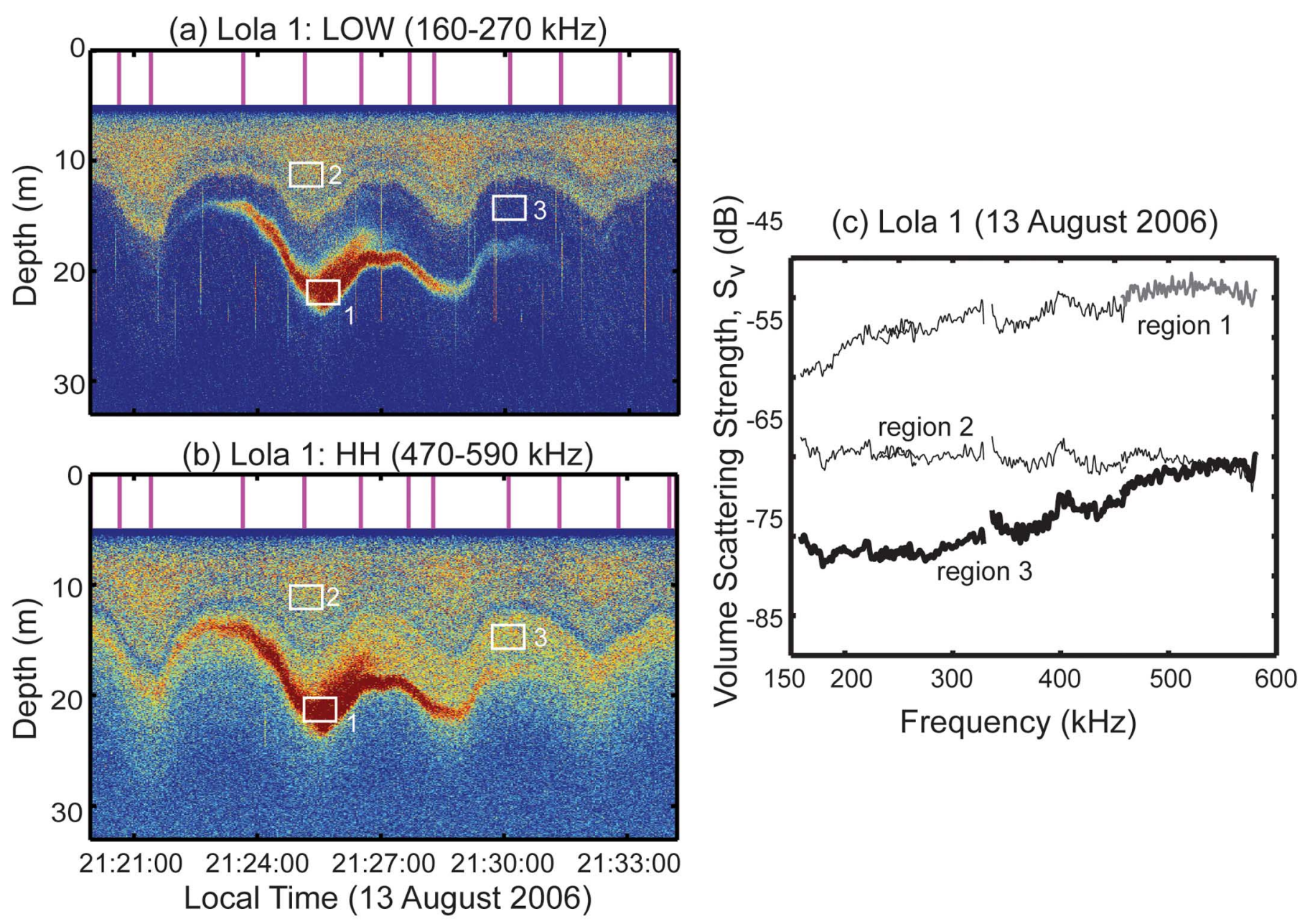

Fig. 8. Compressed pulse output as a function of depth and local time for nonlinear internal wave Lola 1 imaged on August 13, 2006: (a) LOW frequency channel, (b) HH frequency channel, and (c) acoustic spectra associated with the three regions indicated on the compressed pulse output images.

For these inversions, it was assumed that the scattering was dominated by a single type of zooplankton of a single size class. Though this is a great oversimplification, discussed in detail in [15], it is the simplest approach given the fact that the zooplankton net tows were not coincident in space or time. Table I gives the mean sizes and standard deviations of dominant scattering organisms for each multiple opening/closing net and environmental sampling system (MOCNESS) net tow.

Simple least squares inversions for $\varepsilon$ and $\chi_{T}$ were performed in regions where the acoustic spectra were categorized as decreasing across the full bandwidth available. All other parameters do not vary significantly relative to the variations in $\chi_{T}, \chi_{S}$, and $\varepsilon$, which can vary by many orders of magnitude, and thus all other parameters were assumed constant. In fact, though $\chi_{S}$ varied significantly, $\chi_{S} / \chi_{T}$ was generally much smaller than 1 for the data analyzed here, and so the inversions were relatively insensitive to the value of this parameter, within a broad range of values. For the inversions presented here, $\chi_{S} / \chi_{T}$ was assumed to be constant and equal to $1 / 60$, based on the mean value of $\chi_{S}$ in the regions investigated. Inversions were also relatively insensitive to being performed in linear or log space.

Inversions were not performed for any spectra that were not classified as either strictly increasing or decreasing across the full frequency band available, using the criteria outlined in the preceding section.

\section{BIOLOGICAL AND PHYSICAL GROUNDTRUTHING}

To accurately interpret the measured acoustic backscattering, it is necessary to perform both biological and physical groundtruthing. Unlike many previous studies, which are limited by either not having coincident physical groundtruthing or insufficient narrowband acoustic frequencies, almost coincident microstructure and broadband acoustic backscattering measurements were obtained in this study. Details of the direct zooplankton and microstructure measurements and key results pertinent to the interpretation of the acoustic backscattering returns are presented here.

\section{A. Zooplankton}

Five oblique depth-resolved zooplankton net tows were performed with a 1-m² MOCNESS [30]. The zooplankton net sampling system was deployed from the same starboard J-frame as the Edgetech broadband system, precluding the possibility of coincident zooplankton and broadband acoustic sampling. All the zooplankton net tows were performed during daylight hours. The first net tow (MOC 1) was performed in deeper waters off the continental shelf and results of this tow are not pertinent to the work presented here. The remaining four net tows (MOCs 2-5), performed on July 31, and August 10, 24, and 26, 2006, 
(a)

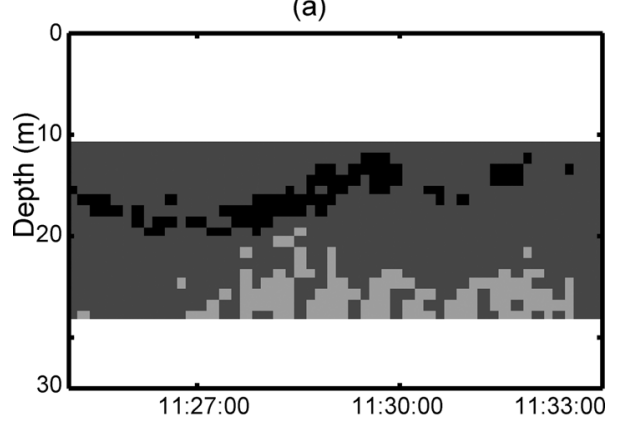

(b)

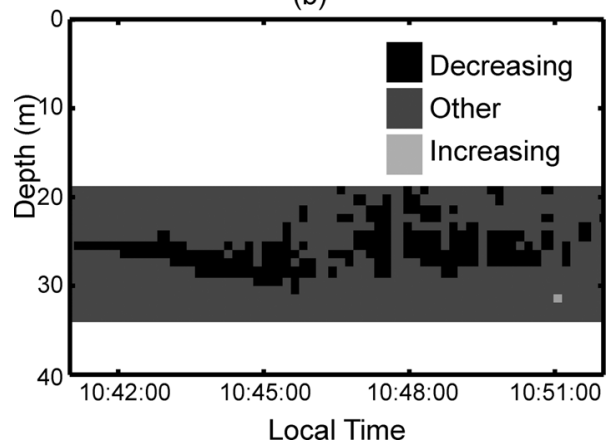

Fig. 9. Acoustic spectra contained in the white boxes in Figs. 6 and 7 for internal waves (a) Holly 17/Isaac 10 and (b) Wyatt 7, imaged on August 10 and 22,2006 , respectively, were calculated and classified according to the scheme in Section III-B. It can be seen that the spectra classified as decreasing are generally associated with the Kelvin-Helmholtz shear instability.

have been fully analyzed (see Table I for summary of pertinent information) for zooplankton composition, size, and abundance. The net tows were performed at locations approximately spanning the across-shelf track of the internal waves tracked. Details of the net tow methods and analysis can be found in [15] and [20]. It should be emphasized that only a restricted number of net tows were performed and these were not coincident in space or time with the broadband measurements presented in this study. This, in combination with inherent zooplankton patchiness and the well-known problems associated with zooplankton avoidance and destruction of fragile animals by net tows, results in the zooplankton groundtruthing data only being used as a rough guide for interpreting the backscattering measurements.

\section{B. Microstructure}

The free-falling microstructure profiler Chameleon [31] was deployed from the fantail, while the broadband acoustic scattering system was simultaneously deployed from the starboard J-frame. The direct microstructure measurements (Fig. 4) resulted in inferences of parameters important for determining scattering, such as, temperature and salinity gradients, the dissipation rate of turbulent kinetic energy $\varepsilon$, and the dissipation rate of temperature variance $\chi_{T}$. Details of the analysis can be found in [31].

\section{ACOUSTIC OBSERVATIONS}

High-resolution images of broadband acoustic backscattering from multiple nonlinear internal wave trains were collected at different stages of their evolution. Highlights of these observations are presented.

\section{A. Scattering Associated With Shear Instabilities}

Turbulence can be generated by the growth of instabilities on highly strained interfaces [7]. Using a single-frequency echosounder and an ADCP, Moum et al. [7] have observed high scattering levels associated with shear instabilities, particularly apparent in the troughs, trailing edges, and wakes of nonlinear internal waves. This was also observed in the present study with the broadband acoustic backscattering system. In fact, many scattering features with structures consistent with distinct Kelvin-Helmholtz shear instabilities were observed acoustically (Figs. 5-7) at significantly greater horizontal resolution than with the direct microstructure measurements.

\section{B. Scattering From Zooplankton}

Scattering spectra consistent with scattering from small zooplankton were observed on multiple occasions [Fig. 5(c)], typically at depths 5-10 $\mathrm{m}$ below the upper mixed layer, and with an apparently diffuse appearance compared to the scattering consistent with microstructure. This scattering layer is generally consistent with the MOCNESS observations of elevated abundance and biomass of small zooplankton, specifically copepods and pteropods. In addition to his commonly observed deeper scattering layer, particularly strong scattering features, also consistent with scattering from biology, were occasionally observed, for example, during the passage of internal wave Lola 1 on August 13, 2006 (Fig. 8). In addition to the shape of the spectra for Lola 1 not being consistent with scattering dominated by microstructure [Fig. 8(c)], this time period was not associated with particularly elevated dissipation rates and the predicted scattering from microstructure could not account for the observed scattering. Thus, it was concluded that the strong scattering feature observed during Lola 1 was associated with a patch of zooplankton. Though the predicted scattering based on the zooplankton net tows could account for the general shape of the spectra observed during Lola 1, it could not account for the high levels of scattering. This discrepancy is attributed to zooplankton patchiness and insufficient biological groundtruthing. One of the features observed during Lola 1 that was also a common observation throughout this study was the approximately flat, or slightly decreasing, acoustic spectrum typically associated with the upper mixed layer.

\section{ACOUSTIC SPECTRA}

A number of distinct frequency-dependent scattering spectra were observed during this experiment (Figs. 5-8). Internal wave Mika 1 imaged on August 14, 2006 illustrates two distinct spectra typical of the observations made throughout this study [Fig. 5(c)]: one that decreases with increasing acoustic frequency, associated with the Kelvin-Helmholtz shear instability with high turbulence levels (region 1), and the other which monotonically increases with increasing acoustic frequency, consistent with scattering from small zooplankton (region 2).

Acoustic spectra were typically calculated by averaging individual spectra over $10 \mathrm{~s}$ and using 1-m depth bins. The depth bins were chosen to match the direct microstructure 
measurements, however, the data could be reanalyzed and the size of the depth bins significantly reduced, the theoretical limit set be the inverse bandwidth of the signal. In future field experiments, the acoustic ping rate could also be increased, which would allow the horizontal scale of the acoustic estimates to be improved, depending on the transducer beamwidth and range. For a number of Kelvin-Helmholtz instabilities, e.g., Holly 17/Isaac 10 (imaged on August 10, 2006) and Wyatt 7 (imaged on August 22, 2006), acoustic spectra were calculated on a fine grid (Fig. 9). The spectrum for each grid point was categorized according to the criteria outlined in Section II-B1, that is, increasing (light gray in Fig. 9), decreasing (black in Fig. 9), or other (dark gray in Fig. 9). It was found that, in contrast to the surrounding spectra, the acoustic scattering spectra associated with these Kelvin-Helmholtz instabilities were decreasing in level with increasing acoustic frequency, consistent with scattering dominated by microstructure.

\section{INFERENCE OF BIOLOGICAL AND PHYSICAL PARAMETERS}

The results of least squares inversions of the measured acoustic spectra for physical ( $\varepsilon$ and $\chi_{T}$ ) and biological (abundance and size) parameters are presented next. Inversions were only performed at locations and frequencies at which a single source of scattering dominated. The focus here is on locations in which the predicted scattering was dominated by microstructure. First, the biological groundtruthing measurements were used to determine likely dominant biological scattering sources. Then, the measured spectra consistent with scattering from zooplankton alone were isolated. Likewise, the direct microstructure measurements were used to determine locations with strong predicted scattering from turbulent microstructure. Inversions at these locations were only performed for measured spectra consistent with scattering dominated by turbulent microstructure.

\section{A. Zooplankton}

The forward calculation, following the methods described in [20], in which acoustic scattering models are used together with information obtained from the net tows to predict volume backscattering and identify dominant scatterers, has been performed for MOCs 2-5. The results of the forward calculation for net tows MOC 4 and MOC 5 are described in detail by Lavery et al. [15] and the results for MOC 2 and MOC 3 are briefly summarized here.

In [15], it was found that the predicted scattering for MOC 4 and MOC 5 was dominated at different depths by either copepods or pteropods, particularly at the upper end of the frequency bandwidth available, with a significant contribution to scattering from amphipods at lower frequencies. For MOC 2, the predicted scattering was generally dominated across the frequency band by elastic-shelled pteropods at depths above the pycnocline, and by weakly scattering fluid-like amphipods at depths below the pycnocline (Table I). For MOC 3, elastic-shelled pteropods again dominated the scattering across the entire frequency band at depths above the pycnocline, while below the pycnocline the scattering was generally not dominated by any individual type of zooplankton, though fluid-like copepods and chaetognaths were important. Finally, it was found that very few gas-bearing zooplankton were observed, and consequently, their contribution to scattering was generally very small. In summary, elastic-shelled pteropods were important contributors to scattering, at least at some depths, in all the zooplankton net tows.

Inversions of the measured acoustic spectra for zooplankton size and abundance were performed at a select number of locations in which the acoustic spectra generally increased with increasing acoustic frequency across the entire frequency band available. It was assumed that a single type of zooplankton of a single size class dominated the scattering. Only results from internal wave Mika 1 (Fig. 5) are presented here as additional results pertaining to inversions for zooplankton parameters can be found in [15], and the focus of this paper is on microstructure. The steeply increasing spectrum observed during Mika 1 (region 2) is consistent with scattering from small zooplankton for which the Rayleigh-to-geometric scattering transition has not yet occurred. Based on the zooplankton net tows, a dominant predicted scatterer that fits this criterion is the elasticshelled pteropod. Though small copepods dominated the numerical abundance and biomass of zooplankton at many locations, the forward calculations revealed that they did not dominate the scattering across the entire frequency band of interest. Thus, least squares inversions were performed to determine the size and abundance of pteropods that best reproduced the measured spectra. The acoustically inferred pteropod diameter $(0.78 \mathrm{~mm})$ was larger than the mean pteropod sizes measured in the nets, by approximately a factor of 2 , and the inferred abundance ( 61 individuals $/ \mathrm{m}^{3}$ ) for Mika 1 was in fair agreement with the abundances measured in the net tows (Table I), though there was far more variability in abundance than size, as would be expected due to the natural patchy spatial structure of zooplankton. Abundance is typically more difficult to accurately infer as it is sensitive to the vagaries of calibration and net avoidance. In this case, the slightly larger inferred size could also be due to the assumptions of the inversion (e.g., mono-specific scatterers and single sized), and a bandwidth that does not completely encompass the Rayleigh-to-geometric scattering transition. In fact, the results are surprisingly good considering that the broadband measurements and zooplankton net sampling were not coincident in time (days apart) or space (several kilometers apart).

\section{B. Microstructure}

Inversions of the measured acoustic spectra for dissipation rates of turbulent kinetic energy and temperature variance were performed at a number of locations in which the acoustic spectra generally decreased with increasing acoustic frequency across the entire frequency band available. Details of the inversion results for internal waves Holly 17/Isaac 10 (Fig. 6) and Wyatt 7 (Fig. 7) are presented here.

The first step in the inversions was to calculate the predicted backscattering based on the model described earlier and the direct microstructure measurements. Predictions are presented in Fig. 10 for internal wave Wyatt 7, at $200 \mathrm{kHz}$, the center frequency for the LOW channel, and $500 \mathrm{kHz}$, the center frequency for the $\mathrm{HH}$ channel. The difference between the predicted scattering at these two frequencies provides a crude estimate as to the shape of the spectrum [Fig. 10(c)]. Next, the measured 


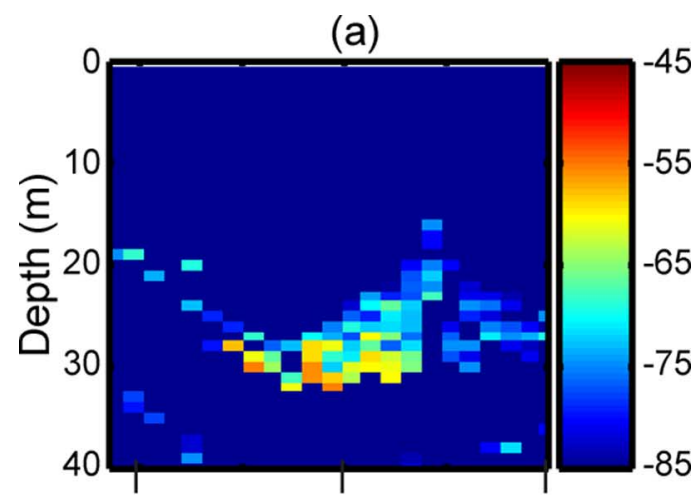

(b)

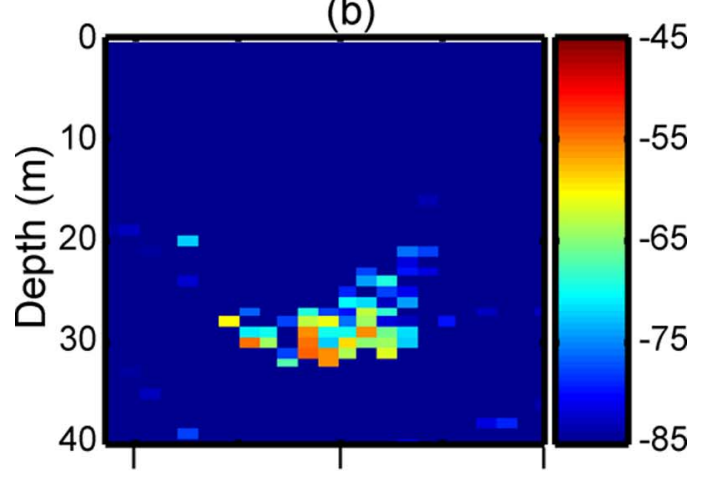

(c)

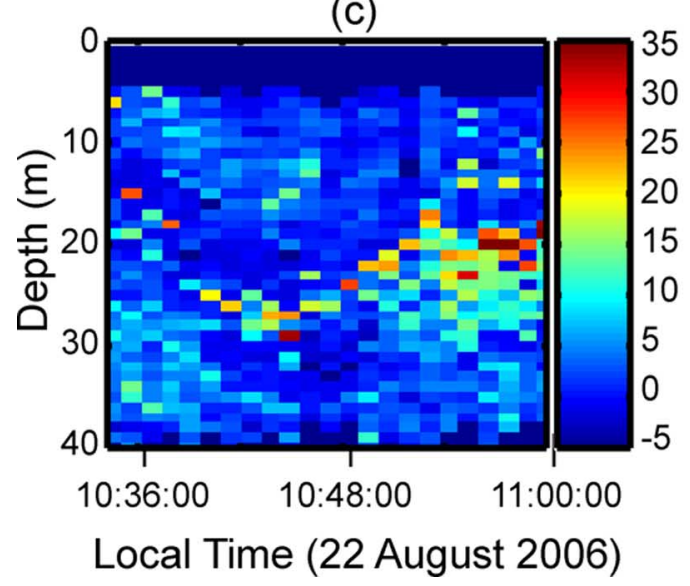

Fig. 10. Predicted volume backscattering strength (in decibels) from microstructure at (a) $200 \mathrm{kHz}$ (LOW channel center frequency) and (b) $500 \mathrm{kHz}$ ( $\mathrm{HH}$ channel center frequency) for internal wave Wyatt 7 imaged on August 22,2006 . The difference in the scattering at 200 and $500 \mathrm{kHz}$ is shown in (c). The predictions are based on (5) and the direct microstructure measurements with no free parameters.

acoustic spectra were classified into one of the three general types as discussed earlier (Fig. 9). As it was observed that the scattering spectra associated with Kelvin-Helmholtz shear instabilities were typically consistent with turbulent microstructure and not zooplankton, that is generally decreasing across the available frequency band, the inversions focus on these regions. In fact, it is highly encouraging that the spectra classified as dominated by turbulent microstructure so closely map regions in which intense turbulence and high-temperature gradients were measured by the direct microstructure instruments.

Only the spectra that were consistent with scattering from turbulent microstructure were used to infer microstructure parameters. The inversion technique was very simple, using a least squares approach to determine best fit values of $\varepsilon$ and $\chi_{T}$. As there were almost coincident microstructure measurements available, it was possible to compare, at least in a qualitative fashion, the direct measurements with the values inferred acoustically. However, due to the scarcity of the direct microstructure profiles relative to the acoustic ping rate (one profile to 200 pings), it was not possible to perform a "point-by-point" comparison.

Though the correlation between the regions in which the spectra classified as dominated by microstructure and regions of high $\varepsilon$ and $\chi_{T}$ is very good, the results of the inversions were less well correlated with the directly measured values (Figs. 11 and 12). The acoustically inferred values of $\varepsilon$ fall well within the range of the directly measured values (Fig. 11), though the directly measured values of $\varepsilon$ vary many more orders of magnitude, suggesting that the statistics of the acoustically inferred values of $\varepsilon$ do not match the statistics of the directly measured values. This was confirmed by looking at histograms of the directly measured and inferred values. This is due to a great extent to the high noise levels of the broadband acoustic system, particularly for the two higher frequency channels (the noise floor is approximately $-72 \mathrm{~dB}$ at $600 \mathrm{kHz}$ ) [15]. To have a decreasing spectrum across the entire frequency band, it is necessary to have very high scattering levels, and thus large values of $\varepsilon$ and $\chi_{T}$.

In addition, as discussed in [15], inversions for microstructure parameters are most robust if either the temperature or salinity dissipation rolloffs, or ideally both, lie within the frequency band available. However, if either (or both) rolloff occurs in the available frequency band, the spectra are most likely not to be decreasing across the entire available frequency band. If the spectra do not decrease across the entire frequency band, it is harder to unambiguously associate these spectra to microstructure alone. The emphasis in this study is on spectra that are unambiguously associated with turbulent microstructure, specifically, decreasing across the entire frequency band, even though the resulting inversions are less robust.

The acoustically inferred values of $\chi_{T}$ are generally larger than those measured directly, though still within the upper range of directly measured values (Fig. 12). Again, this is probably due to the combination of insufficient bandwidth and high acoustic noise levels, and possibly due to the effects of anisotropy [32]. Better agreement between the measured and inferred parameters may follow from a more elaborate classification of the acoustic spectra [32], a greater number of inversions to improve the statistics for comparison with direct measurements, and improved inversion techniques.

\section{SUMMARY AND CONCLUSION}

Nonlinear internal waves play an important role in coastal waters in the transport of sediments, nutrients, zooplankton, and other biological organisms [33], and are often associated with strong mixing events [7]. Many studies have focused on understanding the formation, evolution, and dissipation of nonlinear internal waves in the coastal ocean [7], [34]-[38]. The utility of acoustic scattering techniques in imaging nonlinear internal waves has been previously demonstrated [7], leading to, for example, increased understanding of the evolving structure of 

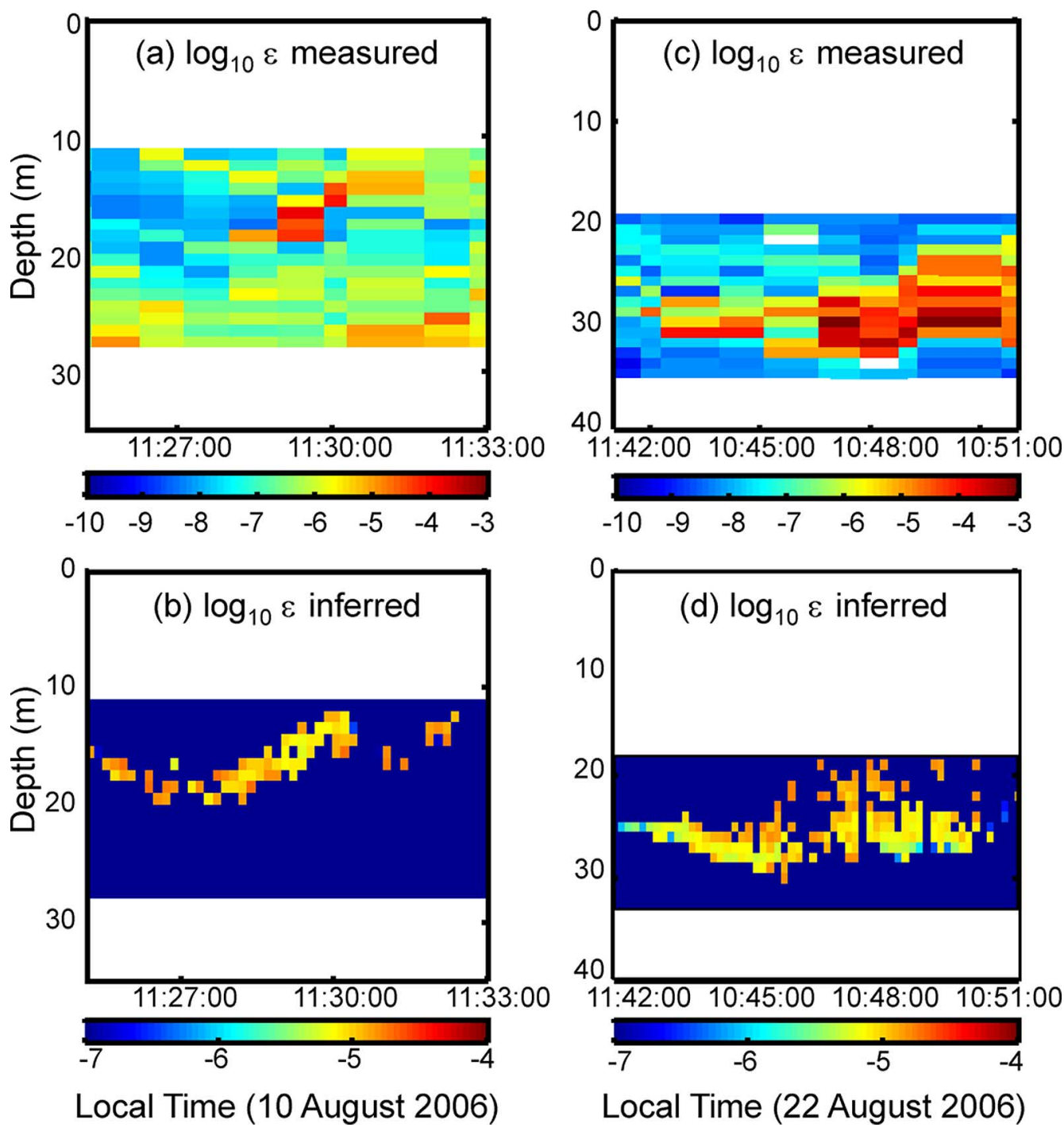

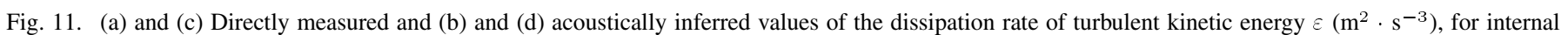
waves (a)-(b) Holly 17/Isaac 10 and (c)-(d) Wyatt 7, imaged on August 10 and 22, 2006, respectively.

the waves as they propagate onshore, and to imaging of the spatial extent of turbulence generation due to shear instabilities. However, previous studies have relied on narrowband acoustic systems with single or multiple frequencies, often without groundtruthing, for which remote quantification of physically meaningful parameters associated with the internal waves was either not possible, ambiguous, or without direct measurements for comparison.

In this study, broadband acoustic scattering techniques have been used for the first time to observe the structure of nonlinear internal waves as they propagate onshore across the continental shelf. Broadband processing has resulted in very high-resolution images of multiple nonlinear internal waves at different stages of their evolution. The broadband content of the acoustic returns has allowed the acoustic backscattering spectra of many scattering features associated with the nonlinear internal waves to be measured. The acoustic spectra have been used to distinguish between scattering dominated by turbulent microstructure and scattering dominated by small zooplankton. In general, it was observed that the scattering was most commonly dominated by zooplankton, except near intensely turbulent Kelvin-Helmholtz shear instabilities, which were almost uniquely associated with acoustic scattering spectra consistent with scattering dominated by microstructure, a very encouraging and suggestive result. Relying heavily on existing scattering models, measured acoustic spectra have been inverted for meaningful biological and physical parameters. The results of these inversions have been compared with measurements from zooplankton net tows and almost-coincident direct microstructure instrumentation.

One of the advantages of broadband acoustic scattering techniques is that they allow meaningful biological and physical parameters to be inferred over spatial scales that cannot be achieved by direct sampling techniques. The range resolution (or vertical resolution for a downward-facing system) for broadband acoustic signals is determined by the inverse bandwidth and the horizontal resolution is determined by the ping rate. In contrast, the typically depth resolution associated with zooplankton net sampling techniques is at best in the range of a few meters to tens of meters, and the nets typically are slowly towed resulting in poor horizontal resolution. Direct 

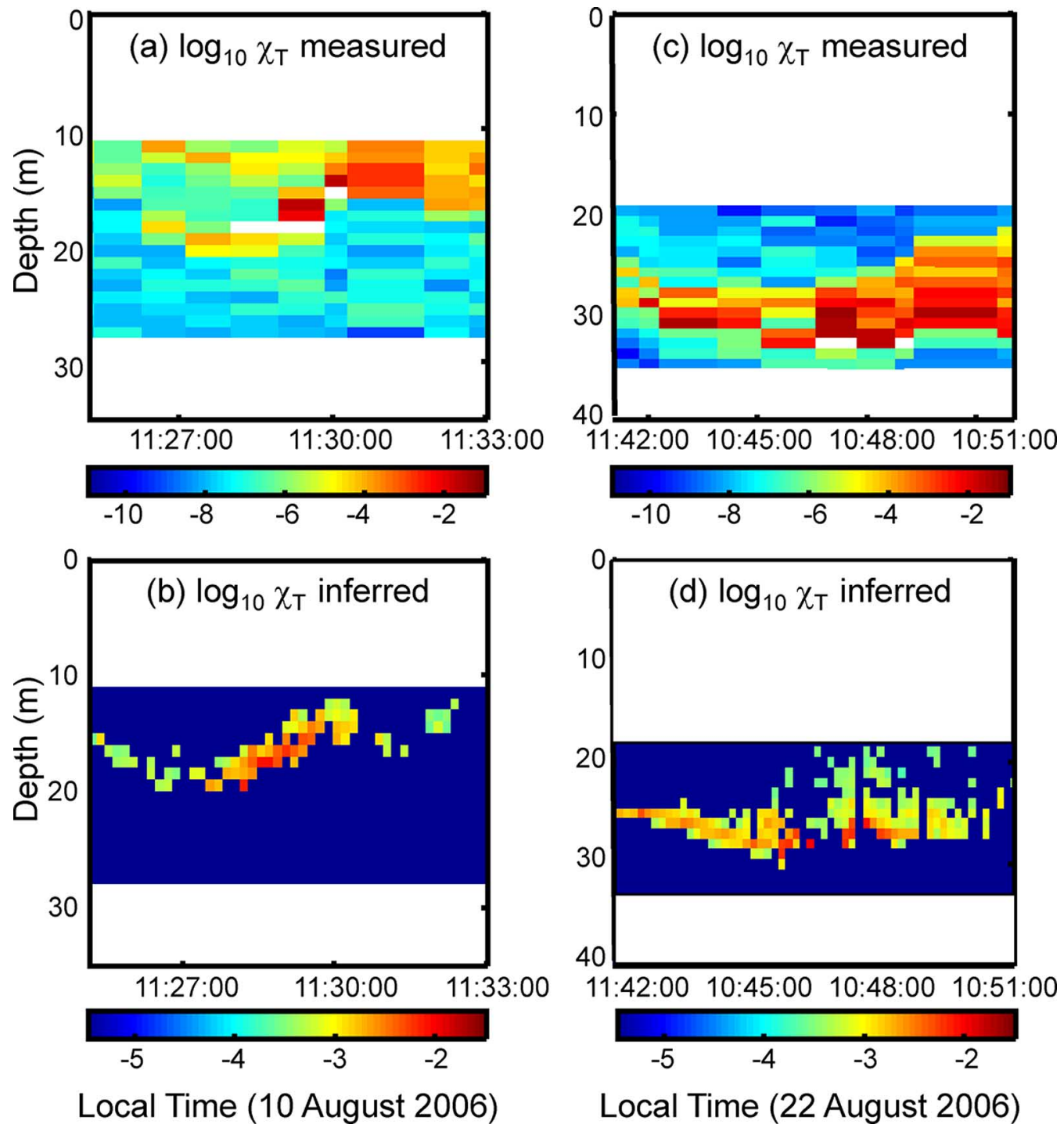

Fig. 12. (a) and (c) Directly measured and (b) and (d) acoustically inferred values of the dissipation rate of temperature variance $\chi_{T}\left(\mathrm{~m}^{2} \cdot \mathrm{s}^{-1}\right)$, for internal waves (a)-(b) Holly 17/Isaac 10 and (c)-(d) Wyatt 7, imaged on August 10 and 22, 2006, respectively.

microstructure measurements provide very high-resolution measurements in one dimension, however the profiles are relatively sparse (one profile every 2-4 min) relative to the acoustic measurements $(1-\mathrm{Hz}$ sampling rate) or the spatial scales of some of the physical features, such as Kelvin-Helmholtz shear instabilities, which were typically only sampled a few times by a microstructure profiler.

Improvements in the interpretation of the measured acoustic spectra could have been achieved with increased bandwidth, as it was not possible to unambiguously interpret many of the measured spectra, for example, regions of high scattering with flat spectra across the available frequency band. It is likely that these spectra arise from a combination of scatterers, however, the possibility of these flat spectra being due to large biological scatterers for which the scattering had already reached the Rayleigh-to-geometric scattering transition could have been addressed with increased bandwidth at the low end of the spectrum. Inversions of the acoustic spectra in regions dominated by turbulent microstructure would also have been more robust with a greater bandwidth, at both ends of the spectrum, as the most unambiguous way to quantify acoustic scattering from microstructure is to measure acoustic scattering spectra over a sufficiently broad frequency band that both the rolloff in the temperature and salinity dissipation spectra are measured. Additional improvements in the interpretation of the measured spectra could have been achieved with increased biological groudthruthing and higher SNRs, particularly for the two higher frequency channels. However, in general, the results presented in this study indicate that broadband acoustic scattering techniques may ultimately be successfully used to quantify physically meaningful parameters, such as dissipation rates of turbulent kinetic energy, in regions of intense turbulence and high temperature and/or salinity gradients.

In the long term, it is hoped that continued development of the techniques outlined here will result in the routine achievement of high-resolution images of nonlinear internal waves, as well as accurate quantification of the broadband backscattering from microstructure generated by nonlinear internal waves, potentially resulting in an important supplemental tool for understanding their energy dissipation mechanisms. 


\section{ACKNOWLEDGMENT}

There are many people the authors would like to thank for making this work possible: K. Fisher, P. Heslinga, and D. Leong for helping collect the acoustic and MOCNESS data during the monthlong experiment, S. Wright and other EdgeTech employees for making the development of this system possible, T. Stanton for assistance with the development of the system, and for lending them his 1000-m coax cable, M. Neeley-Brown and R. Kreth for supporting the Oregon State University (OSU) Ocean Mixing operations, as well as J. Kemp and his team and the captain and crew of the $R / V$ Oceanus.

\section{REFERENCES}

[1] J. R. Proni and J. R. Apel, "On the use of high-frequency acoustics for the study of internal waves and microstructure," J. Geophys. Res., vol. 90, no. 9, pp. 1147-1151, 1975.

[2] T. R. Haury, M. G. Briscoe, and M. H. Orr, "Tidally generated internal wave packets in Massachusetts Bay," Nature, vol. 278, no. 5702, pp. 312-317, 1979.

[3] H. Sandstrom, J. A. Elliott, and N. A. Cochrane, "Observing groups of solitary internal waves and turbulence with BATFISH and echosounder," J. Phys. Oceanogr., vol. 19, no. 7, pp. 987-997, 1989.

[4] M. V. Trevorrow, "Observations of internal solitary waves near the Oregon coast with an inverted echo sounder," J. Geophys. Res. C. Oceans, vol. 103, no. C4, pp. 7671-7680, 1998.

[5] D. M. Farmer and L. Armi, "The generation and trapping of internal solitary waves over topography," Science, vol. 283, pp. 188-190, 1999.

[6] M. H. Orr, T. R. Haury, P. H. Wiene, and M. G. Briscoe, "Backscatter of high-frequency $(200 \mathrm{kHz})$ acoustic wavefields from ocean turbulence," J. Acoust. Soc. Amer., vol. 108, no. 4, pp. 1595-1601, 2000.

[7] J. N. Moum, D. M. Farmer, W. D. Smyth, L. Armi, and S. Vagle, "Structure and generation of turbulence at interfaces strained by internal solitary waves propagation shoreward over the continental shelf," J. Phys. Ocean., vol. 33, no. 10, pp. 2093-2122, 2003.

[8] D. M. Farmer and J. D. Smith, "Tidal interaction of stratified flow with a sill in Knight Inlet," Deep Sea Res., vol. 27A, pp. 239-254, 1979.

[9] P. F. Cummins, L. Armi, and S. Vagle, "Upstream internal hydraulic jumps," J. Phys. Oceanogr., vol. 36, no. 5, pp. 753-769, 2006.

[10] S. Vagle and D. M. Farmer, "The measurement of bubble-size distributions by acoustical backscatter," J. Atmos. Ocean. Technol., vol. 9, pp. 630-644, 1994.

[11] S. A. Thorpe, M. S. Cure, and A. Graham, "Sonar observations of Langmuir circulation and estimation of dispersion of floating particles," $J$. Atmos. Ocean. Technol., vol. 11, pp. 1273-1294, 1994.

[12] H. E. Seim, M. C. Gregg, and R. T. Miyamoto, "Acoustic backscatter from turbulent microstructure," J. Atmos. Ocean. Technol., vol. 12, no. 2, pp. 367-380, 1995.

[13] T. Ross and R. Lueck, "Sound scattering from oceanic turbulence," Geophys. Res. Lett., vol. 30, no. 6, 2003, DOI: 10.1029/ 2002GL016733.

[14] J. D. Warren, T. K. Stanton, P. H. Wiebe, and H. E. Seim, "Inference of biological and physical parameters in an internal wave using multiplefrequency, acoustic-scattering data," ICES J. Mar. Sci., vol. 60, no. 5, pp. 1033-1046, 2003

[15] A. C. Lavery, D. Chu, and J. Moum, "Measurements of scattering from zooplankton and oceanic microstructure using a broadband echosounder," ICES J. Mar. Sci., vol. 67, no. 2, pp. 379-394, 2010.

[16] B. J. Rothschild and T. R. Osborn, "Small-scale turbulence and plankton contact rates," J. Plank. Res., vol. 10, pp. 465-474, 1988

[17] L. Seuront, F. Schmitt, and Y. Lagadeuc, "Turbulence intermittency, small-scale phytoplankton patchiness and encounter rates in plankton: Where do we go from here?," Deep Sea Res. I, vol. 48, no. 5, pp. $1199-1215,2001$.

[18] T. K. Stanton, D. Chu, P. H. Wiebe, L. V. Martin, and R. L. Eastwood, "Sound scattering by several zooplankton groups. I. Experimental determination of dominant scattering mechanisms," J. Acoust. Soc. Amer., vol. 103, no. 1, pp. 225-235, 1998.

[19] T. K. Stanton, D. Chu, and P. H. Wiebe, "Sound scattering by several zooplankton groups. II. Scattering models," J. Acoust. Soc. Amer., vol. 103, no. 1, pp. 236-253, 1998

[20] A. C. Lavery, P. H. Wiebe, T. K. Stanton, G. Lawson, M. C. Benfield, and N. Copley, "Determining dominant scatterers of sound in mixed zooplankton populations," J. Acoust. Soc. Amer., vol. 122, no. 6, pp. 3304-3326, 2007
[21] A. C. Lavery, R. W. Schmitt, and T. K. Stanton, "High-frequency acoustic scattering from turbulent oceanic microstructure: The importance of density fluctuations," J. Acoust. Soc. Amer., vol. 114, no. 5, pp. 2685-2697, 2003.

[22] W. D. Smyth, J. N. Moum, and D. R. Caldwell, "The efficiency of mixing in turbulent patches: Inferences from direct simulations and microstructure observations," J. Phys. Oceanogr., vol. 31, no. 8, pp. 1969-1992, 2001.

[23] D. J. Tang, J. N. Moum, J. F. Lynch, P. Abbot, R. Chapman, P. H. Dahl, T. F. Duda, G. Gawarkiewicz, S. Glenn, J. A. Goff, H. Graber, J. Kemp, A. Maffei, J. D. Nash, and A. Newhall, "Shallow Water '06: A joint acoustic propagation/nonlinear internal wave physics experiment," Oceanography, vol. 20, no. 4, pp. 156-167, 2007.

[24] K. G. Foote and D. N. MacLennan, "Comparison of copper and tungsten carbide calibration spheres," J. Acoust. Soc. Amer., vol. 75, pp. 612-616, 1984.

[25] D. Chu and T. K. Stanton, "Application of pulse compression techniques to broadband acoustic scattering by live individual zooplankton," J. Acoust. Soc. Amer., vol. 104, no. 1, pp. 39-55, 1998.

[26] T. K. Stanton and D. Chu, "Calibration of broadband active acoustic systems using a single standard spherical target," J. Acoust. Soc. Amer., vol. 124, pp. 128-136, 2008.

[27] T. K. Stanton, D. Chu, J. M. Jech, and J. D. Irish, "New broadband methods for resonance classification and high-resolution imagery of fish with swimbladders using a modified commercial broadband echosounder," ICES J. Mar. Sci., vol. 67, no. 2, pp. 365-378, 2010.

[28] T. Ross, C. Garrett, and R. Lueck, "On the turbulent co-spectrum of two scalars and its effect on acoustic scattering from oceanic turbulence," J. Fluid Mech., vol. 514, pp. 107-119, 2004.

[29] N. S. Oakey, "Determination of the rate of dissipation of turbulent energy from simultaneous temperature and shear microstructure measurements," J. Phys. Oceanogr., vol. 12, pp. 256-271, 1982.

[30] P. H. Wiebe, A. W. Morton, A. M. Bradley, R. H. Backus, J. E. Craddock, V. Barber, T. J. Cowles, and G. R. Flierl, "New developments in the MOCNESS, An apparatus for sampling zooplankton and micronekton," Mar. Biol., vol. 87, no. 3, pp. 313-323, 1985.

[31] J. N. Moum, M. C. Gregg, R. C. Lien, and M. E. Carr, "Comparison of turbulent kinetic energy dissipation rate estimates from two ocean microstructure profilers," J. Atmos. Ocean. Technol., vol. 12, pp. 346-366, 1995.

[32] D. Leong, "Assessing the isotropy of ocean turbulence using broadband acoustics," M.Sc. thesis, Dept. Oceanogr., Dalhousie Univ., Halifax, NS, Canada, 2009.

[33] J. Pineda, "Predictable upwelling and the shoreward transport of planktonic larvae by internal tidal bores," Science, vol. 253, no. 5019, pp. 548-551, 1991.

[34] E. L. Shroyer, J. N. Moum, and J. D. Nash, "Observations of polarity reversal in shoaling nonlinear internal waves," J. Phys. Oceanogr., vol. 39, no. 3, pp. 671-701, 2009.

[35] J. M. Klymak and J. N. Moum, "Internal solitary waves of elevation advancing on a shoaling shelf," Geophys. Res. Lett., vol. 30, no. 20, p. 2045, 2003, DOI: 10.1029/2003GL017706.

[36] A. Scotti, R. Beardsley, and B. Butman, "On the interpretation of energy and energy fluxes of nonlinear internal waves: An example from Massachusetts Bay," J. Fluid Mech., vol. 561, pp. 103-112, 2006.

[37] J. N. Moum, D. M. Farmer, E. L. Shroyer, W. D. Smyth, and L. Armi, "Dissipative losses in nonlinear internal waves propagating across the continental shelf," J. Phys. Oceanogr., vol. 37, no. 7, pp. 1989-1995, 2007.

[38] J. N. Moum, J. M. Klymak, J. D. Nash, A. Perlin, and W. D. Smyth, "Energy transport by nonlinear internal waves," J. Phys. Oceanogr., vol. 37, no. 7, pp. 1968-1988, 2007

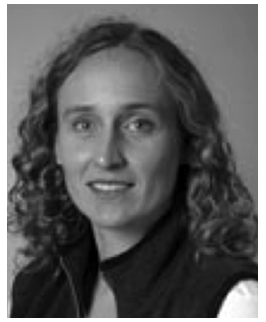

Andone C. Lavery received the B.A. degree in mathematics from Cambridge University, Cambridge, U.K., in 1991 and the M.Sc. and Ph.D. degrees in physics from Cornell University, Ithaca, NY, in 1995 and 1999, respectively.

She was a Postdoctoral Scholar from 1999 to 2001 with the Woods Hole Oceanographic Institution (WHOI), Woods Hole, MA, and an Office of Naval Research (ONR) Ocean Acoustics Postdoctoral Fellow from 2001 to 2002, also at WHOI. Since 2002, she has been a Member of the Scientific Staff, Department of Applied Ocean Physics and Engineering, WHOI. Her research interests include high-frequency acoustic scattering and propagation in discrete and random media, developing physics-based acoustic scattering models for marine organisms and small scale fluid processes, performing laboratory measurements for validating scattering models, developing instruments and 
signal processing methods for ocean measurements of scattering from biologics and physical processes.

Dr. Lavery is a member of the Acoustical Society of America, the Oceanography Society, and the American Physical Society. She is currently Associate Editor for the Journal of the Acoustical Society of America Express Letters.

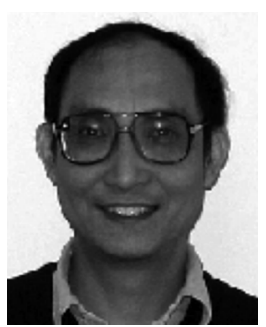

Dezhang Chu received the B.A. degree in electrical engineering from the China University of Geosciences, Wuhan, China, in 1982 and the Ph.D. degree in geophysics from the University of Wisconsin, Madison, in 1989.

He was a Postdoctoral Scholar from 1989 to 1990 and a Postdoctoral Investigator from 1990 to 1991 with the Woods Hole Oceanographic Institution (WHOI), Woods Hole, MA. From 1991 to 2007, he was first a Research Associate and then a Research Specialist with the Department of Applied Ocean Physics and Engineering, WHOI. During that period at WHOI, he developed acoustic scattering models for zooplankton, fish, and seafloor, conducted laboratory and ocean measurements of scattering, as well as developed signal processing methods for acoustic scattering by marine organisms. Since 2007 he has been with the Acoustics Team of the NOAA/NMFS Northwest Fisheries Science Center (NWFSC), Fishery Resources Analysis and Monitoring Division, Seattle, WA. His research currently is focused on acoustic scattering, characterization, and classification of fish.

Dr. Chu is a Fellow of the Acoustical Society of America.

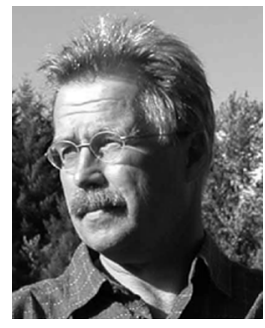

James N. Moum received the B.Sc. and M.Sc. degrees in engineering from the University of Toronto, Toronto, ON, Canada, in 1978 and 1979, respectively, and the Ph.D. degree in physics from the University of British Columbia, Vancouver, BC, Canada, in 1984.

Since 1984, he has worked at the College of Oceanic and Atmospheric Sciences, Oregon State University, Corvallis, studying small scale oceanic fluid dynamics and their effects on turbulence and mixing of the ocean. He has been a Full Professor at Oregon State University since 1996. 